\title{
Cell-free DNA from cerebrospinal fluid can be used to detect the EGFR mutation status of lung adenocarcinoma patients with central nervous system metastasis
}

\author{
Yang Liu ${ }^{1 \#}$, Sen Yang ${ }^{2,3 \#}$, Jiuzhou Zhao ${ }^{4}$, Zhen $\mathrm{He}^{2}$, Jie $\mathrm{Ma}^{4}$, Yongjun Guo ${ }^{4}$, Wei Wang ${ }^{5}$, Akihiko Yoshizawa ${ }^{6}$, \\ Arsela Prelaj ${ }^{7,8}$, Marcello Tiseo ${ }^{9}$, Nicola Normanno ${ }^{10}$, Paul E. Van Schil ${ }^{11}$, Qiming Wang ${ }^{2}$, Xiaopeng Yang ${ }^{3}$ \\ ${ }^{1}$ Department of Radiotherapy, Affiliated Cancer Hospital of Zhengzhou University, Henan Cancer Hospital, Zhengzhou, China; ${ }^{2}$ Department of \\ Internal Medicine, Affiliated Cancer Hospital of Zhengzhou University, Henan Cancer Hospital, Zhengzhou, China; ${ }^{3}$ Department of Neurology, \\ The Second Affiliated Hospital of Zhengzhou University, Zhengzhou, China; ${ }^{4}$ Department of Molecular Pathology, Affiliated Cancer Hospital of \\ Zhengzhou University, Henan Cancer Hospital, Henan Key Laboratory of Molecular Pathology, Zhengzhou, China; ${ }^{5}$ Henan Medical Association, \\ Zhengzhou, China; ${ }^{6}$ Department of Diagnostic Pathology, Kyoto University Hospital, Sakyo-ku, Kyoto, Japan; ${ }^{7}$ Medical Oncology Department, \\ Fondazione IRCCS Istituto Nazionale Tumori, Milan, Italy; ${ }^{8}$ Department of Electronics, Information, and Bioengineering, Polytechnic University of \\ Milan, Milan, Italy; ${ }^{9}$ Department of Medicine and Surgery, University of Parma and Medical Oncology Unit, University Hospital of Parma, Parma, \\ Italy; ${ }^{10}$ Cell Biology and Biotherapy Unit, Istituto Nazionale Tumori-IRCCS_-"Fondazione G. Pascale”, Naples, Italy; ${ }^{11}$ Department of Thoracic \\ and Vascular Surgery, University Hospital of Antwerp, Edegem, Belgiu \\ Contributions: (I) Conception and design: Q Wang, X Yang; (II) Administrative support: W Wang, Y Guo; (III) Provision of study materials or \\ patients: Z He, Y Liu, J Ma; (IV) Collection and assembly of data: S Yang, J Zhao; (V) Data analysis and interpretation: S Yang; (VI) Manuscript \\ writing: All authors; (VII) Final approval of manuscript: All authors. \\ \#These authors contributed equally to this work. \\ Correspondence to: Qiming Wang. Department of Internal Medicine, Affiliated Cancer Hospital of Zhengzhou University, Henan Cancer Hospital, \\ Zhengzhou, China. Email: qimingwang1006@126.com; Xiaopeng Yang. Department of Neurology, The Second Affiliated Hospital of Zhengzhou \\ University, Zhengzhou, China. Email: yaxipe39@126.com.
}

Background: EGFR tyrosine kinase inhibitors (TKIs) have revolutionized the therapeutic approach for EGFR mutated patients. However, acquired resistance to EGFR-TKI therapy is unavoidable. Repeat biopsy cannot be used, and peripheral blood detection shows a low positive rate in cases of brain-only disease progression.

Methods: Droplet digital polymerase chain reaction (PCR) (ddPCR) was performed on the plasma and cerebrospinal fluid (CSF) samples of 79 lung adenocarcinoma (LUAD) patients with EGFR mutations and central nervous system (CNS) metastasis. The differences in the EGFR mutation status between the paired plasma and CSF samples were assessed, and the role of CSF testing as a predictor of overall survival was evaluated.

Results: The CSF of patients with neurological symptoms, EGFR-TKI treatment, or leptomeningeal metastasis (LM) had a significantly higher positive rate of EGFR mutation compared to the plasma samples $(\mathrm{P}=0.001, \mathrm{P}=0.035, \mathrm{P}=0.019$, respectively). Moreover, EGFR mutation status in CSF was consistent with neurological symptoms and LM (kappa $=0.455, \mathrm{P}<0.001$; kappa $=0.508, \mathrm{P}<0.001$; respectively). For the patients with brain metastasis, EGFR mutation-positive rate in CSF samples was lower than that in plasma samples $(28.3 \%$ vs. $64.2 \%, \mathrm{P}<0.001)$, while the patients with $\mathrm{LM}$ had the opposite result $(84.6 \%$ vs. $38.5 \%$, $\mathrm{P}=0.004)$. Moreover, patients with EGFR mutation in their CSF experienced worse survival [hazard ratio (HR) $=2.93,95 \%$ confidence interval (CI): 1.45-5.92; $\left.\mathrm{P}=0.003, \mathrm{P}_{\text {adjust }}<0.0001\right]$.

Conclusions: The EGFR mutation status of CSF was different from that of plasma and is correlated with patient prognosis. CSF could be helpful in detecting the EGFR mutation status of patients, particularly in cases of LM. 
Keywords: Non-small cell lung cancer (NSCLC); leptomeningeal metastasis (LM); liquid biopsy; cerebrospinal fluid (CSF); EGFR mutation

Submitted Jul 30, 2020. Accepted for publication Feb 20, 2021.

doi: $10.21037 /$ tlcr-21-62

View this article at: http://dx.doi.org/10.21037/tlcr-21-62

\section{Introduction}

Lung cancer, with the highest rate of cancer-related mortality worldwide (1), is among the most common human malignancies. Non-small cell lung cancer (NSCLC) accounts for approximately $90 \%$ of all global lung cancer cases (2). Epidermal growth factor receptor (EGFR)activating mutations, such as L858R and exon 19 deletion (E19Del), have been found in approximately $10-15 \%$ of NSCLC patients in Western countries and 40-55\% of patients in Asian countries (3). NSCLC patients with EGFR mutations have a significantly high risk of developing metastasis of the central nervous system (CNS), including leptomeningeal metastasis (LM) and brain metastasis (BM), which is associated with poor prognosis and compromised quality of life (4). The combined incidence of BM and LM in NSCLC patients is more than $50 \%(5-7)$. For NSCLC patients with $\mathrm{BM}$ and $\mathrm{LM}$ who receive EGFR tyrosine kinase inhibitor (TKI) therapy, the median overall survival (OS) is 10.3-16.2 months (8) and 4.5-11 months $(9,10)$, respectively, which is markedly shorter than that for patients without CNS metastasis. EGFR-TKIs have ushered in a new era of targeted therapy in NSCLC and have dramatically improved the progression-free survival (PFS) of patients who harbor EGFR-activating mutations (11-17). Nevertheless, acquired resistance to EGFR-TKI therapy is inevitable. Therefore, rebiopsy is needed to detect secondary resistance mechanisms in order to develop a new treatment strategy.

With its many advantages, the detection of tumor-specific genetic alterations in body fluids has become a supplement to or even a replacement for established tissue-based tumor diagnostics, and thus has garnered considerable attention in academic research and the medical testing industry (18). Moreover, circulating tumor DNA (ctDNA) has been widely used to detect EGFR mutations status in patients who develop resistance to targeted therapy (19). ctDNA derives from tumors or circulating tumor cells (CTCs), and constitutes a fraction of the cell-free DNA (cfDNA) that is extracted from body fluids, which also contain DNA derived from non-transformed cells (20). ctDNA has been shown to be capable of accurately reflecting tumor-specific genomic alterations and can be used to monitor tumor progression, response to treatment, and relapse (21). Therefore, ctDNA testing might be an ideal detection tool in cancer patients. Moreover, cerebrospinal fluid (CSF) has shown utility as a liquid biopsy medium for the gene expression profiling of NSCLC patients with LM, contributing to more sensitive and effective diagnoses (22). However, due to the bloodbrain barrier (BBB), the EGFR mutation status in CSF is not consistent with that in plasma, and controversy still surrounds which of the two is a better indicator of EGFR alterations in patients with CNS metastasis $(23,24)$. Several studies have reported tumor-associated alterations being detectable in the CSF ctDNA of patients with various primary or metastatic brain tumors, while little ctDNA was found in the plasma of these patients (25-28). These findings indicate that CSF has a stronger capability to recapitulate the genomic profile of CNS tumors/metastatic sites than does plasma.

In the present study, we performed droplet digital polymerase chain reaction (ddPCR) on paired plasma and CSF samples from 79 lung adenocarcinoma (LUAD) patients with $E G F R$ mutations and CNS metastasis. Through comparing the EGFR mutation status of the plasma and CSF samples, we aimed to determine the ability of CSF compared to plasma in detecting EGFR mutation in patients with LUAD and CNS metastasis.

We present the following article in accordance with the MDAR reporting checklist (available at http://dx.doi. org/10.21037/tlcr-21-62).

\section{Methods}

\section{Patients}

This study included 79 LUAD patients with CNS metastasis harboring EGFR mutations in tumor tissue (L858R or E19Del). All patients were diagnosed at Henan Cancer Hospital between 2012 and 2018. The last follow-up took 
Table 1 Characteristics of 79 patients with brain metastases and leptomeningeal metastases

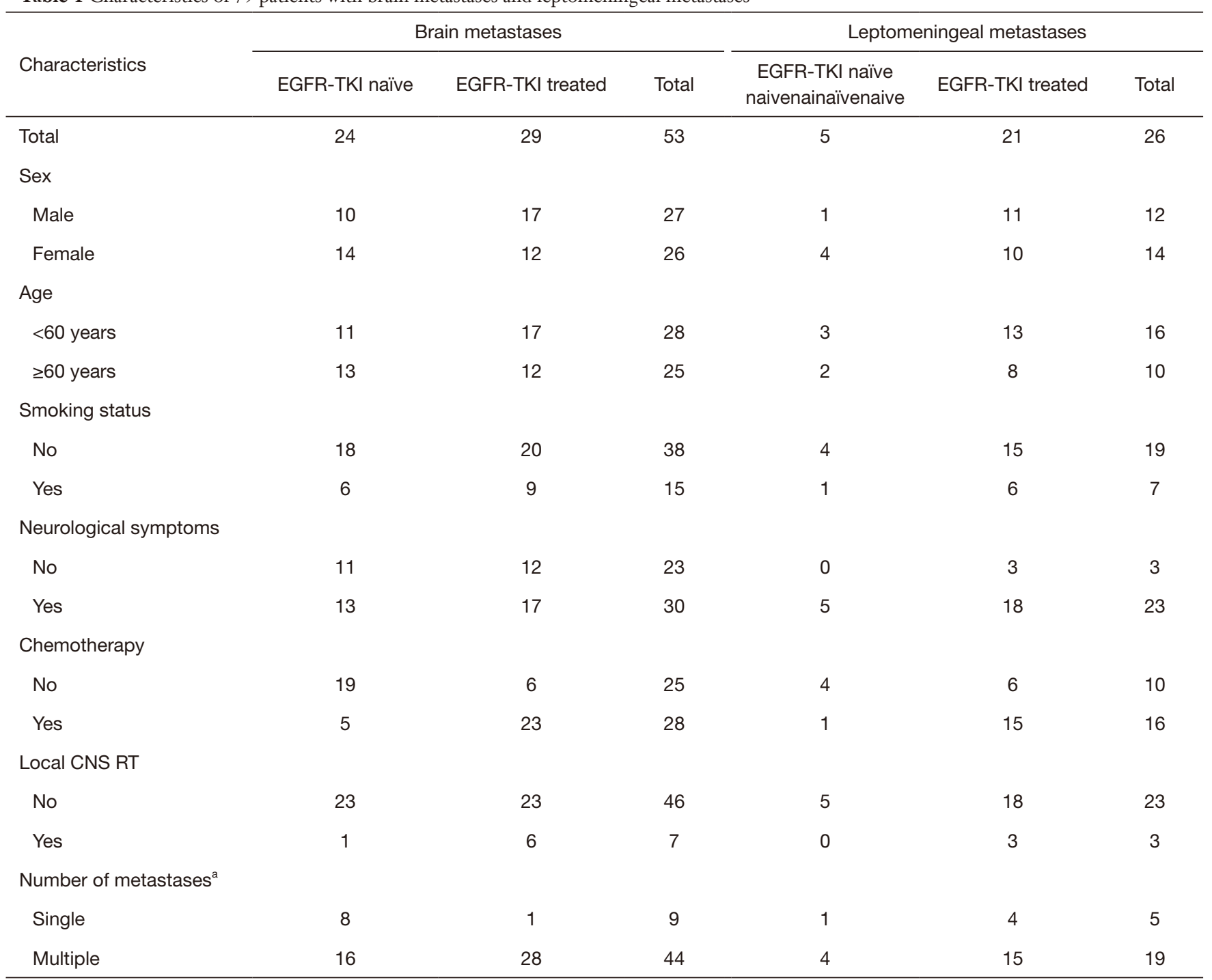

${ }^{a}$, two patients with leptomeningeal metastases and EGFR-TKI treatment were negative by MRI but positive by ThinPrep cytologic test. TKI, tyrosine kinase inhibitors; CNS, central nervous system; RT, radiotherapy.

place on April 20, 2019, and six patients were lost to followup. CNS metastasis was confirmed in all patients by brain magnetic resonance imaging (MRI) and ThinPrep cytologic testing (TCT), which were performed according to standard procedures by experienced radiologists and pathologists. Patient clinical data including sex, age, smoking status, chemotherapy, local CNS radiotherapy, and history of EGFR-TKI treatment were collected from the electronic medical records of Henan Cancer Hospital (Table 1).

All procedures performed in this study involving human participants were in accordance with the Declaration of Helsinki (as revised in 2013). This study was conducted in accordance with the ethical guidelines of the United States' common rule, and the protocol was approved by the Research Ethics Committee of Henan Cancer Hospital. All patients signed informed consent forms.

\section{Sample processing and DNA extraction}

After the CNS metastases were diagnosed, CSF $(10 \mathrm{~mL})$ and peripheral blood $(15 \mathrm{~mL})$ were collected by lumbar puncture and blood sampling respectively (Figure 1). The collected samples were preserved in EDTA tubes (SANLI) for the isolation of cfDNA. The DNA isolation procedure 


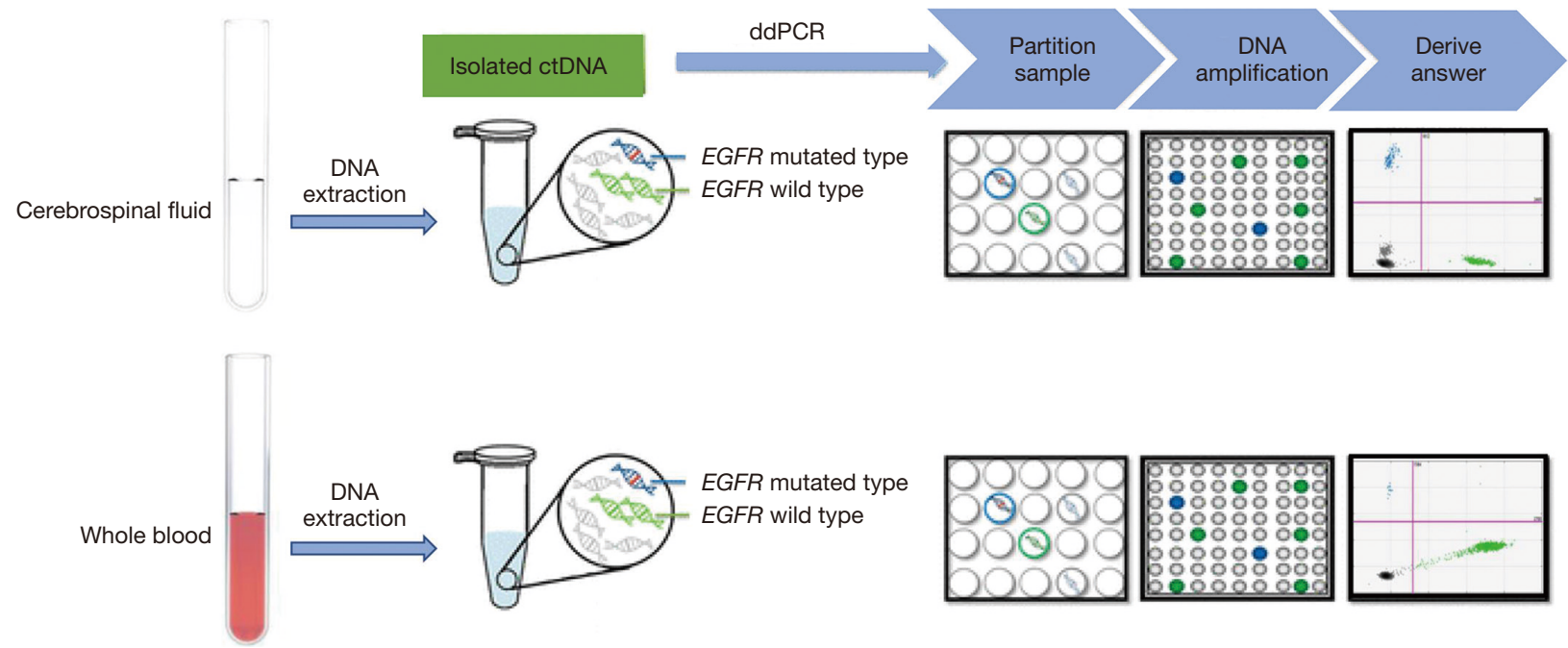

Figure 1 The workflow of the present study. ctDNA, circulating tumor DNA; ddPCR, droplet digital polymerase chain reaction.

was performed within $2 \mathrm{~h}$ after sample collection. To collect plasma, the peripheral blood samples were subjected to centrifugation. Then, the plasma and CSF were first centrifuged at 2,000 $\times \mathrm{g}$ for $10 \mathrm{~min}$ at $4{ }^{\circ} \mathrm{C}$ and again at $16,000 \times \mathrm{g}$ for $10 \mathrm{~min}$ at $4{ }^{\circ} \mathrm{C}$. The QIAamp Circulating Nucleic Acid Kit (\#55114, QIAGEN, Hilden, Germany) was used to capture cfDNA according to the manufacturer's instructions. The quality and concentration of the DNA were assessed using a Qubit dsDNA HS assay (\#1204008450, Life Technologies, Carlsbad, CA, USA). DNA was also isolated from the CSF sediment for the ddPCR test. The extracted DNA was stored at $-20^{\circ} \mathrm{C}$ for later analysis.

\section{ddPCR test}

A QX200 digital PCR system (Bio-Rad Laboratories, Hercules, CA, USA) was used to conduct the ddPCR assays (29). For exon 19 deletions, p.L858R and p.T790M were detected using a human-EGFR ddPCR detection kit (\#CB240005, YuanQi) according to the manufacturer's instructions. The PCR program was as follows: $95^{\circ} \mathrm{C}$ for $10 \mathrm{~min} ; 40$ cycles of $94^{\circ} \mathrm{C}$ for $15 \mathrm{~s}$ and $58^{\circ} \mathrm{C}$ for $60 \mathrm{~s} ; 98^{\circ} \mathrm{C}$ for $10 \mathrm{~min}$; and $4{ }^{\circ} \mathrm{C}$ for $5 \mathrm{~min}$. The reaction volume was set to $40 \mu \mathrm{L}$. The reaction temperature was changed at a rate of less than $2{ }^{\circ} \mathrm{C} / \mathrm{s}$. After thermal cycling, the amplified samples were loaded into a BioRad reader (\#771BR24449, BioRad Laboratories) for quantification. These experiments were performed according to the protocols of the Mutation Detection Best Practices Guidelines supplied by Bio-Rad Laboratories (Figure 1).

\section{Statistical analysis}

Paired $\chi^{2}$ (McNemar's test) and kappa tests were utilized to compare the clinical characteristics and EGFR mutation status in the plasma and CSF samples from the enrolled patients, and to compare the different subgroups divided according to their clinical data. Wilcoxon signed-rank test was used to evaluate the difference in EGFR mutation abundance between paired CSF and plasma samples. The patients' survival probabilities associated with the EGFR mutation status of CSF were analyzed by KaplanMeier curves and adjusted with clinical variables. Receiver operating characteristic (ROC) curve and time-dependent area under the curve (AUC) analyses were used to evaluate the accuracy of the predictive model by integrating the effects of both clinical variables and the EGFR mutation status of CSF on patient survival. All statistical analyses were performed using SPSS 20.0 (IBM Corp., Armonk, NY, USA), with the level of statistical significance set at a $\mathrm{P}$ value $<0.05$, unless otherwise indicated.

\section{Results}

\section{Patient characteristics}

The 79 patients enrolled in this study were diagnosed with advanced LUAD and confirmed to harbor sensitizing EGFR mutations, with 25 of these patients being treatment naïve. As shown in Table 1, 53 patients were diagnosed with BM and 26 with LM. Furthermore, 50 had a history of EGFRTKI treatment, 44 patients had received chemotherapy, and 
10 had received local CNS radiotherapy. The median age of the patients was 56 (range, 29-76) years, and 39 patients were male, including 22 who had history of smoking; none of the female patients smoked. Most patients (53 of 79) had neurological symptoms, including dizziness and headache, and most (63 of 77) had more than one metastatic lesion of the CNS.

\section{EGFR gene mutation status in CSF supernatant and sediment}

First, to determine whether supernatant or sediment could better represent CSF, McNemar's test and kappa tests were used to compare the EGFR mutation status between paired samples of CSF supernatant and sediment from the 79 patients. As shown in Table S1, no statistically significant differences were observed in the positive rate of $E G F R$ mutations between the supernatant and sediment of CSF (43.0\% vs. $34.2 \%, \mathrm{P}=0.092$ ). Moreover, the two samples were generally consistent (kappa $=0.656$; range, $0.40-0.75$; $\mathrm{P}<0.001)$. However, a difference was observed in the abundance (mutated alleles/mutated alleles and wild type alleles) of positive supernatant and sediment samples, with the abundance in the supernatant higher than that in the sediment [median (quartile): $33.00 \%(23.00-49.30 \%)$ and $5.30 \%(2.20-15.00 \%)$, respectively].

\section{EGFR gene mutation status and clinical characteristics}

To determine if there was any correlation between EGFR mutation and clinical features, we compared the EGFR mutation status of the patients' plasma and CSF samples with various clinical characteristics. As shown in Table 2, a higher frequency of EGFR mutation was observed in the plasma samples of never smokers, patients with multiple CNS metastases, patients treated with radiotherapy, and patients without $\mathrm{LM}(61.4 \%$ vs. $40.9 \%, \mathrm{P}=0.002 ; 58.0 \%$ vs. $40.0 \%, \mathrm{P}<0.001 ; 58.7 \%$ vs. $50.0 \%, \mathrm{P}=0.001$; and $64.2 \%$ vs. $38.5 \%, \mathrm{P}=0.015$; respectively). No statistically significant differences in EGFR mutation status were found for other variables including sex, age, neurological symptoms, chemotherapy treatment history, or EGFT-TKI treatment history $(\mathrm{P}>0.05)$. All these characteristics showed poor consistency with EGFR mutation status in plasma (kappa $<0.4)$.

For the CSF samples, patients with neurological symptoms (50.9\% vs. $36.4 \%, \mathrm{P}=0.032)$, no history of radiotherapy $(47.8 \%$ vs. $40.0 \%, \mathrm{P}<0.001)$, presence of multiple CNS metastases (including LM) (47.6\% vs. 35.7\%, $\mathrm{P}<0.001)$, and an EGFT-TKI treatment history $(54.0 \%$ vs. $34.5 \%, \mathrm{P}=0.035)$, along with non-smokers $(64.2 \%$ vs. $11.5 \%, \mathrm{P}=0.001)$ and $\mathrm{LM}$ patients $(84.6 \%$ vs. $28.3 \%$, $\mathrm{P}=0.019)$, had a higher risk of being EGFR mutationpositive. The other variables had no significant correlation with the EGFR mutation status of CSF. Moreover, neurological symptoms and LM showed general consistency with $E G F R$ mutation status in $\mathrm{CSF}$ (kappa $=0.455, \mathrm{P}<0.001$; kappa $=0.508, \mathrm{P}<0.001$; respectively).

\section{EGFR gene mutation status in CSF and plasma samples in different subgroups}

To ascertain the difference between CSF and plasma in reflecting the EGFR mutations of patients, the EGFR mutation status between paired CSF and plasma samples from all patients was compared. As shown in Table S2, there was not a statistically significant difference in the positive rate of $E G F R$ mutations between the paired CSF and plasma samples $(\mathrm{P}=0.360$; kappa $=-0.081, \mathrm{P}=0.466)$. However, the number of positive CSF samples was significantly higher than that of plasma samples $[33.00 \%(16.80-49.30 \%)$ and $2.05 \%(0.67-14.75 \%)$, respectively].

Next, the 79 patients were divided into different subgroups according to their clinical characteristics. The EGFR mutation status of the paired CSF and plasma samples from patients in different subgroups was compared. In the BM patient group, the positive rate of EGFR mutations was notably lower in the CSF samples than in plasma samples $(28.3 \%$ vs. $64.2 \%, \mathrm{P}<0.001$; $\mathrm{kappa}=0.093$, $\mathrm{P}=0.381$ ), while the opposite result was observed in the $\mathrm{LM}$ patient group $(84.6 \%$ vs. $38.5 \%, \mathrm{P}=0.004$; kappa $=-0.061$, $\mathrm{P}=0.606$ ) (Tables $\mathrm{S} 3, \mathrm{~S} 4)$. No significant difference between the EGFR mutation-positive rate of the CSF and plasma samples was observed in other subgroups, including patients with neurological symptoms ( $\mathrm{P}=0.697)$, without EGFR-TKI treatment history $(\mathrm{P}=0.202)$, with EGFR-TKI treatment history $(\mathrm{P}=0.777)$, with EGFR E19Del $(\mathrm{P}=0.821)$ and EGFR 21 exon L858R mutation $(\mathrm{P}=0.320)$ (Tables $\mathrm{S} 5-\mathrm{S} 10)$.

\section{Different subtypes of EGFR gene mutations in CSF and plasma}

Although the positive rates of E19Del and p.L858R mutation were not significantly different between the CSF and plasma samples from any of the patients, as shown in Tables S9,S10, respectively, we questioned whether the 
Table 2 Comparison of EGFR mutation status of the patients with different characteristics from plasma and CSF samples of 79 patients

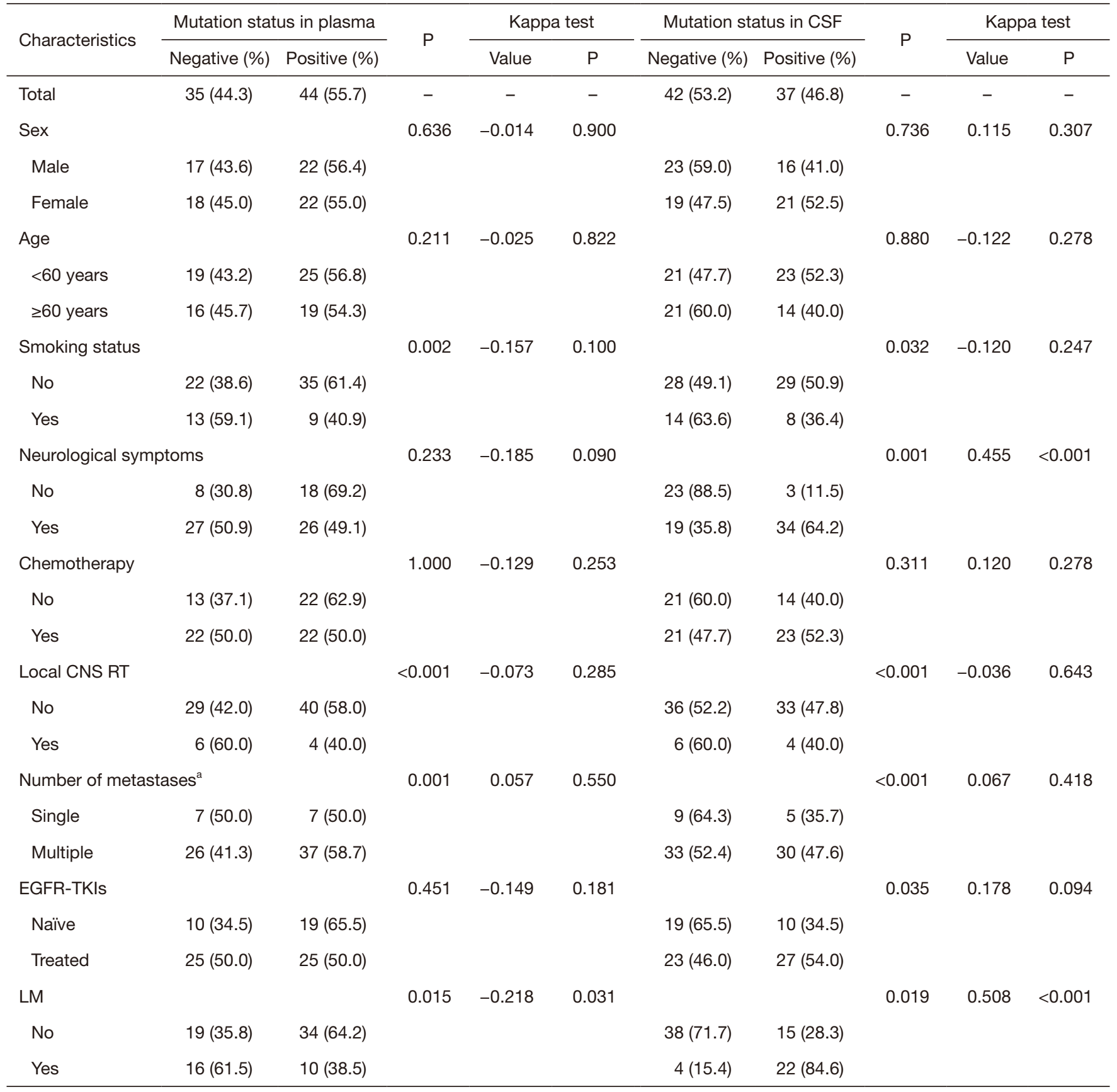

${ }^{a}$, two patients with leptomeningeal metastases and EGFR-TKI treatment were negative by MRI but positive by ThinPrep cytologic test. CSF, cerebrospinal fluid; TKI, tyrosine kinase inhibitors; CNS, central nervous system; RT, radiotherapy; LM, leptomeningeal metastasis.

EGFR mutation status differed between the plasma and CSF samples of patients with BM or LM. Here, three mutations were analyzed: p.L858R in exon 21, E19Dels, and p.T790M in exon 20). As shown in Table 3, the positive rates of L858R, E19Dels, and p.T790M mutations in the
CSF samples from BM patients were all lower than those in the plasma samples. However, for patients with LM, the positive rates of p.L858R and E19Dels in the CSF samples were both higher than those in the plasma samples, while T790M showed no significant difference. 
Table 3 The EGFR mutation types in plasma and cerebrospinal fluid among patients with CNS metastasis

\begin{tabular}{|c|c|c|c|c|c|c|}
\hline Characteristics & \multicolumn{3}{|c|}{ Plasma } & \multicolumn{3}{|c|}{ Cerebrospinal fluid } \\
\hline \multicolumn{7}{|l|}{ Brain metastases } \\
\hline EGFR-TKI naïve & $7 / 10(70.0 \%)$ & $11 / 14(78.6 \%)$ & $1 / 1(100.0 \%)$ & $1 / 10(10.0 \%)$ & $4 / 14(28.6 \%)$ & $0 / 1(00.0 \%)$ \\
\hline EGFR-TKI treated ${ }^{a}$ & $7 / 15(46.7 \%)$ & $10 / 15(66.7 \%)$ & 4/7 (57.1\%) & 4/15 (26.7\%) & $7 / 15(46.7 \%)$ & $0 / 7(00.0 \%)$ \\
\hline \multicolumn{7}{|c|}{ Leptomeningeal metastases } \\
\hline EGFR-TKI naïve ${ }^{b}$ & 0/3 (00.0\%) & 1/3 (33.3\%) & 0 & $3 / 3(100.0 \%)$ & $3 / 3(100.0 \%)$ & 0 \\
\hline EGFR-TKI treated & $5 / 14(35.7 .0 \%)$ & 4/7 (57.1\%) & $4 / 9$ (44.4\%) & $11 / 14(78.6 \%)$ & $6 / 7(85.7 \%)$ & $4 / 9(44.4 \%)$ \\
\hline Total & $5 / 17$ (29.4\%) & $5 / 10(50.0 \%)$ & $4 / 9(44.4 \%)$ & $14 / 17$ (82.4\%) & 9/10 (90.0\%) & $4 / 9(44.4 \%)$ \\
\hline
\end{tabular}

a 1 patient had both L858R and 19del EGFR mutation; ${ }^{\mathrm{b}}, 1$ patient had both L858R and 19del EGFR mutation. TKI, tyrosine kinase inhibitors; CNS, central nervous system.

\section{EGFR gene mutation abundance in CSF and plasma}

Since ddPCR allows for the quantitative detection of ctDNA, a Wilcoxon signed-rank test was employed to assess the differences in EGFR mutation abundance in paired CSF and plasma samples in positive patients. The median EGFR mutation abundances in CSF and plasma samples were $33.50 \%$ and $1.75 \%$, respectively, with a median difference of 29.14\%. The Wilcoxon signed-rank test results $(Z=-3.509$ and $\mathrm{P}<0.001)$ indicated that the EGFR mutation abundance in CSF was significantly higher than that in plasma (Table S11). The comparison of the median EGFR mutation abundance in the paired CSF and plasma samples from patients with BM and LM is displayed in Figure 2, including three specific mutations: p.L858R in exon 21, E19dels, and exon 20 p.T790M. Overall, in LM patients, the abundance of E19dels, p.L858R, and p.T790M in CSF was higher than that in plasma, while in BM patients, the abundance of p.T790M in CSF was lower than that in plasma.

\section{EGFR gene mutation status and patient survival}

Finally, we investigated if there was any correlation between EGFR mutation status and OS. Complete follow-up data of 73 of 79 patients were available for analysis. The patients' plasma and CSF samples were separately analyzed using Kaplan-Meier survival analysis, with adjustment for available covariations including sex, age, and smoking status. As shown in Figure $3 \mathrm{~A}$, the analysis of plasma samples indicated that the difference in median survival time between patients with and without EGFR mutations was not significant [19.7 vs. 14.4 months, hazard ratio (HR) $=1.24,95 \%$, confidence interval $(\mathrm{CI}): 0.63-2.45 ; \mathrm{P}=0.531$, $\left.\mathrm{P}_{\text {adjust }}=0.819\right]$. However, patients with EGFR mutation in their CSF had significantly worse survival than patients without $E G F R$ mutation in their $\mathrm{CSF}(\mathrm{HR}=2.93,95 \% \mathrm{CI}$ : 1.45-5.92; $\mathrm{P}=0.003, \mathrm{P}_{\text {adjust }}<0.0001$ ) (Figure $3 B$ ).

Then, we assessed the role of the EGFR mutation status of CSF in predicting the OS of patients by using timedependent AUC and ROC curves at the $12^{\text {th }}$ month (or 1-year survival). Compared with the models for age, sex, and smoking status, the time-dependent AUC plotted for additional EGFR mutation status in CSF was improved. The AUCs were improved from 70.81-82.40\% ( $\mathrm{P}=0.066)$ for 1-year survival (Figure 3C,D).

\section{Discussion}

In this study, ddPCR was used to quantificationally detect EGFR mutation status in paired plasma and CSF samples from LUAD patients with CNS metastasis. The results showed that for patients with LM, the abundance of EGFR mutation in CSF EGFR mutation-positive samples was higher than that in plasma, and the EGFR mutation status of CSF was significantly different from that of plasma. Further survival analysis indicated that patients with EGFR mutation in their CSF had worse survival than patients without EGFR mutation in their CSF. These results suggest that for patients with LM, EGFR mutations can be detected more effectively in CSF than in plasma samples. This presents a potentially effective alternative or supplement for 

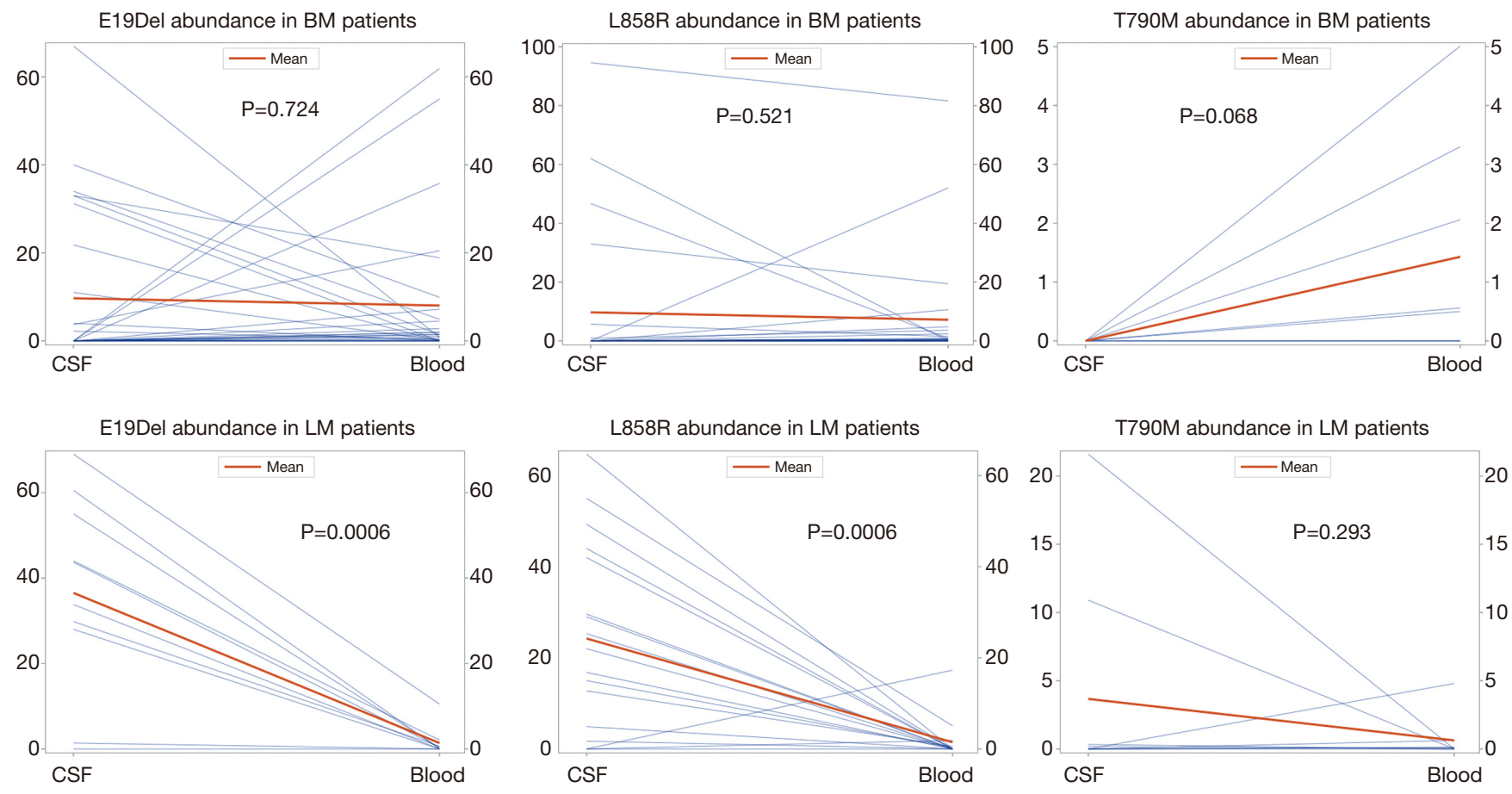

Figure 2 Distribution of different EGFR mutation sites in paired plasma and CSF. In leptomeningeal metastasis patients, the abundance of E19del, L858R, and T790M in CSF was higher than that in plasma, while the abundance of T790M in CSF was lower than that in plasma. $\mathrm{BM}$, brain metastasis; LM, leptomeningeal metastasis; CSF, cerebrospinal fluid.

plasma liquid biopsy for the detection of EGFR mutations in patients with CNS metastasis. Furthermore, the EGFR mutation status of CSF could serve as a biomarker for predicting the OS of patients with CNS metastasis.

Next-generation sequencing (NGS) can be used to monitor LM development and guide precision medicine (4). However, NGS has not been widely used in clinical practice due to its high cost and technological complexity. ddPCR was used as our liquid biopsy method over SuperARMS and NGS due to its higher sensitivity and specificity (the sensitivity is lower than $0.1 \%$ ), which suggests that more patients would benefit from this method.

Most patients harboring EGFR mutations develop CNS metastasis (30); however, treatment with EGFR-TKIs eventually results in acquired resistance. The acquired resistance mechanisms can be either EGFR-dependent or EGFR-independent, such as is in T790M or MET amplification, respectively. The latter mechanism seems to be the most frequent, appearing in nearly $50 \%$ of $\mathrm{LM}$ cases (31). Therefore, the treatment strategy selected depends on the patient's EGFR mutation status at a particular time. Liquid biopsy facilitates the detection of the EGFR mutation status of patients who are unwilling to undergo tissue aspiration biopsy or from whom a tumor tissue sample cannot be obtained (32). cfDNA is a short fragment (usually 130-180 base pairs) double-stranded DNA that is present in plasma and other body fluids (33-36). It is thought to originate mainly from apoptotic or necrotic cell death, although active release mechanisms have also been investigated $(37,38)$. In cancer patients, cfDNA is derived from both non-malignant and malignant cells, with the percentage of ctDNA originating from cancer cells ranging from $3 \%$ to $93 \%$ (38). Moreover, cancer patients are frequently observed to have increased levels of cfDNA in their plasma, and under certain circumstances, increased cfDNA is considered to be an adverse prognostic factor (39). Increased levels of cfDNA might also be attributable to impaired renal clearance function or production of white plasma cells (WBC) $(40,41)$. In contrast, ctDNA in CSF is quite stable, meaning it has the potential to facilitate and supplement the diagnosis of LM due to the low sensitivity of CSF cytology, especially in cases that cannot be detected by traditional cytopathological analysis (26).

We included both EGFR-TKI-naïve and EGFR-TKItreated patients in this study. In the EGFR-TKI-naïve patients, the EGFR mutation-positive rate in plasma was 

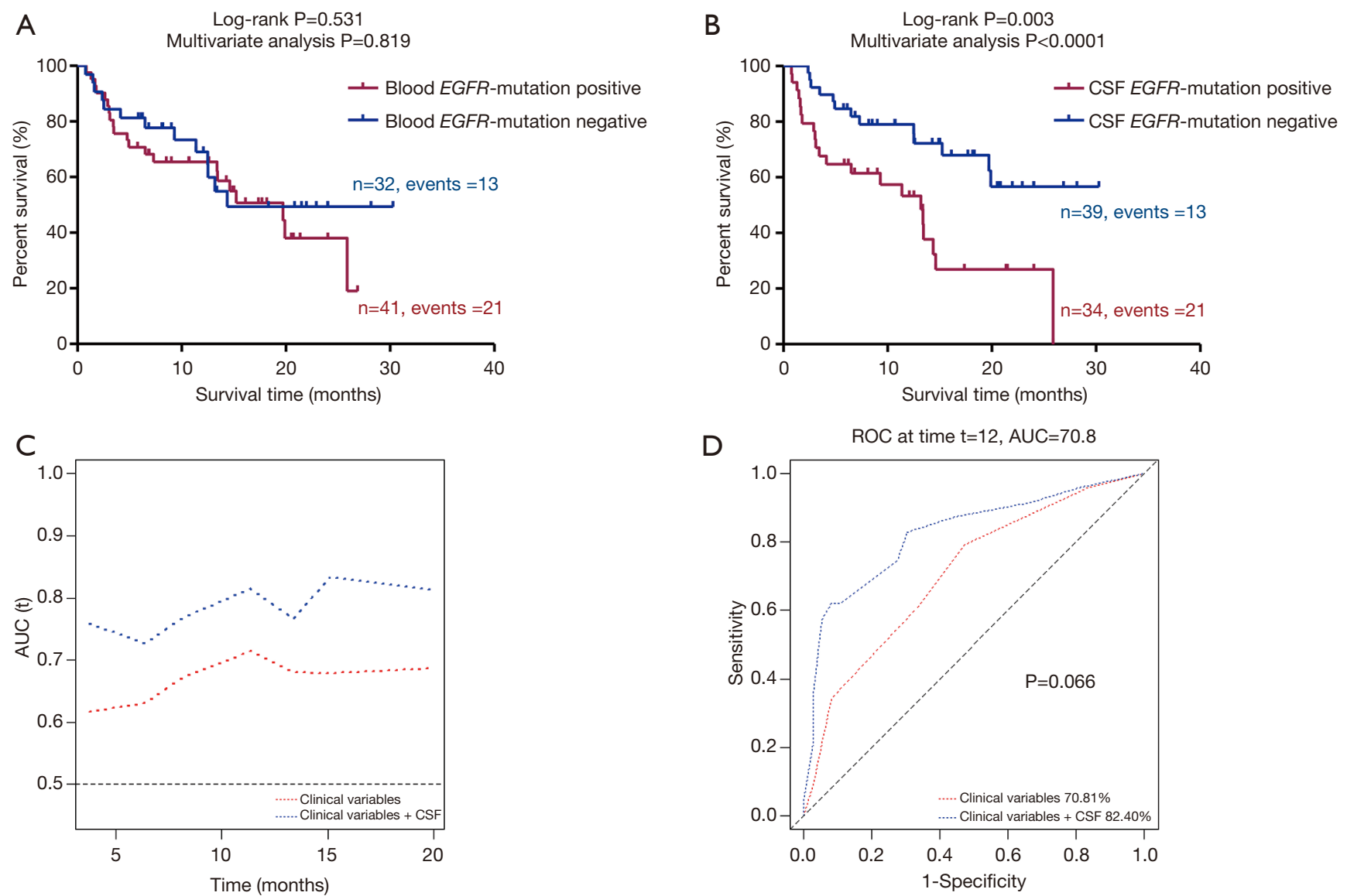

Figure 3 Kaplan-Meier survival curve according to EGFR mutation status in plasma and CSF. The difference of median survival time between patients with and without EGFR mutations was not significant (A). The patients with EGFR mutations in their CSF had significantly worse survival than patients without EGFR mutation in their CSF (B). Compared with the model for age, sex, and smoking status, the model with addition of EGFR mutation status in CSF showed improvement of time-dependent AUC plot (C,D). CSF, cerebrospinal fluid; AUC, area under the curve.

$65.5 \%$, which is generally consistent with previous reports. However, a downward trend in the EGFR mutation-positive rate was observed in the plasma of patients with a history of EGFR-TKI therapy. This can be explained by the fact that few EGFR mutation-positive NSCLC cells survive in extracranial circulations after EGFR-TKI treatment. Nevertheless, the EGFR mutation-positive rate in the CSF samples displayed an opposite trend. The reason for this might be that leptomeninges and CSF could become a safe harbor for protecting EGFR mutation-positive NSCLC cells during EGFR-TKI therapy because of the BBB.

In this study, although no statistically significant difference or consistency was observed in the positive rate of EGFR mutations in CSF and plasma samples when the entire cohort was analyzed, for patients with neurological symptoms, LM, or those with an EGFR-TKI treatment history, EGFR mutation-positive rates in CSF were higher than those in plasma. Moreover, in the patients with LM, the mutation abundance in CSF was significantly higher than that in the plasma samples. This might be due to ctDNA only constituting a small fraction of the cfDNA in plasma arising from the large amount of DNA produced by normal cells, while few normal cells exist in CSF, which results in a notably higher proportion of ctDNA. These results suggest that for patients with EGFR-TKI resistance and CNS metastasis, testing CSF-derived cfDNA might be more effective than testing plasma. Furthermore, our analysis indicated that patients with EGFR mutations in their CSF had worse survival, meaning that meningeal metastases might have occurred in these patients, which 
would dramatically increase the risk of death, although this was not diagnosed by TCT or MRI.

The mechanisms of cfDNA release are poorly understood, and their predictive role and relationship to tumor burden are controversial and still being investigated $(42,43)$. We assessed the correlation between the OS of patients and the cfDNA status in both plasma and CSF samples, and found that the cfDNA status in CSF was negatively correlated with patient survival. This result suggests that EGFR mutation status or cfDNA in the CSF might be a promising predictor for OS in LUAD patients with CNS metastasis. Previous studies demonstrated a correlation between plasma levels of ctDNA and survival $(21,22)$. The lack of correlation found in this study could be due to the fact that plasma is not adequate to predict patients' survival for patients with CNS metastases.

As far as we know, the present study is the largest to explore EGFR mutation status in the CSF of LUAD patients with CNS metastasis, and has yielded new insight into the comparison of EGFR mutation status in paired CSF and plasma samples with ddPCR. Nevertheless, this study has some limitations. First, the sample size of 79 patients was not large enough, which might have resulted in the introduction of biases, including low statistical power, higher false discovery rate, and low reproducibility. Secondly, only L858R, E19del, and T790M mutations can be detected by ddPCR, which limited the exploration of the possible molecular mechanism of BM. Finally, this study was performed in a Chinese population and, therefore, may not be representative of other ethnic groups.

In conclusion, we found that the EGFR mutation status in CSF was different from that in plasma, and CSF could more effectively reflect the EGFR mutation status of LUAD patients with CNS metastasis. Our results indicate that the detection of EGFR mutation status in CSF could serve as an efficient alternative or supplement to plasma testing. Furthermore, for patients with acquired resistance to EGFR-TKIs, liquid biopsy of CSF would be an excellent tool for detecting EGFR mutation status to inform future treatment decision-making.

\section{Acknowledgments}

Marcello Tiseo is currently supported by the Associazione Italiana per la Ricerca sul Cancro (AIRC) under Investigator Grant (IG) No. IG2017-20074.

Funding: This study was supported by the Henan Province Health and Youth Subject Leader Training Project
$\{[2020] 60\}$; the Leading Talent Cultivation Project of Henan Health Science and Technology Innovation Talents (YXKC2020009); the Zhongyuan Qianren Jihua (ZYQR201912118); the Henan International Joint Laboratory of drug resisitance and reversal of targeted therapy for lung cancer $\{[2021] 10\}$; the Henan Medical Key Laboratory of Refractory lung cancer $\{[2020] 27\}$; the Henan Refractory Lung Cancer Drug Treatment Engineering Technology Research Center $\{[2020] 4\}$; the 51282 Project Leading Talent of Henan Provincial Health Science and Technology Innovation Talents $\{[2016] 32\}$; the Huilan Charity Fund (HL-HS2020-129); Major public welfare projects in Henan Province (201300310400).

\section{Footnote}

Reporting Checklist: The authors have completed the MDAR reporting checklist. Available at http://dx.doi.org/10.21037/ tlcr-21-62

Data Sharing Statement: Available at http://dx.doi. org/10.21037/tlcr-21-62

Conflicts of Interest: All authors have completed the ICMJE uniform disclosure form (available at http://dx.doi. org/10.21037/tlcr-21-62). MT has received speakers' and consultants' fees from Astra-Zeneca, Pfizer, Eli-Lilly, BMS, Novartis, Roche, MSD, Boehringer Ingelheim, Otsuka, Takeda, and Pierre Fabre. MT received institutional research grants from Astra-Zeneca and Boehringer Ingelheim, during the conduct of the study; PEVS is an external expert of National Cancer Institute (France), MSD, AstraZeneca (institutional fees only), during the conduct of the study; NN received speakers' and consultants' fee from MSD, Qiagen, Bayer, Biocartis, Illumina, Incyte, Roche, BMS, MERCK, Thermofisher, Boehringer Ingelheim, Astrazeneca, Sanofi, Eli Lilly, Nicola Normanno received institutional research grants from MERCK, Sysmex, Thermofisher, QIAGEN, Roche, Astrazeneca, Biocartis, Illumina, during the conduct of the study. The other authors have no conflicts of interest to declare.

Ethical Statement: The authors are accountable for all aspects of the work in ensuring that questions related to the accuracy or integrity of any part of the work are appropriately investigated and resolved. All procedures performed in this study involving human participants were in accordance with the Declaration of Helsinki (as revised 
in 2013). This study was conducted in accordance with the ethical guidelines of the United States' common rule, and the protocol was approved by the Research Ethics Committee of Henan Cancer Hospital. All the patients in the present study have signed informed consent.

Open Access Statement: This is an Open Access article distributed in accordance with the Creative Commons Attribution-NonCommercial-NoDerivs 4.0 International License (CC BY-NC-ND 4.0), which permits the noncommercial replication and distribution of the article with the strict proviso that no changes or edits are made and the original work is properly cited (including links to both the formal publication through the relevant DOI and the license). See: https://creativecommons.org/licenses/by-nc-nd/4.0/.

\section{References}

1. Siegel RL, Miller KD, Jemal A. Cancer statistics, 2020. CA Cancer J Clin 2020;70:7-30.

2. Goldstraw P, Ball D, Jett JR, et al. Non-small-cell lung cancer. Lancet 2011;378:1727-40.

3. Kosaka T, Yatabe Y, Endoh H, et al. Mutations of the epidermal growth factor receptor gene in lung cancer: biological and clinical implications. Cancer Res 2004;64:8919-23.

4. Fan Y, Zhu X, Xu Y, et al. Cell-Cycle and DNA-Damage Response Pathway Is Involved in Leptomeningeal Metastasis of Non-Small Cell Lung Cancer. Clin Cancer Res 2018;24:209-16.

5. Palma JA, Fernandez-Torron R, Esteve-Belloch P, et al. Leptomeningeal carcinomatosis: prognostic value of clinical, cerebrospinal fluid, and neuroimaging features. Clin Neurol Neurosurg 2013;115:19-25.

6. Choong NW, Dietrich S, Seiwert TY, et al. Gefitinib response of erlotinib-refractory lung cancer involving meninges--role of EGFR mutation. Nat Clin Pract Oncol 2006;3:50-7; quiz $1 \mathrm{p}$ following 57.

7. Rangachari D, Yamaguchi N, VanderLaan PA, et al. Brain metastases in patients with EGFR-mutated or ALKrearranged non-small-cell lung cancers. Lung Cancer 2015;88:108-11.

8. Fan Y, Xu X, Xie C. EGFR-TKI therapy for patients with brain metastases from non-small-cell lung cancer: a pooled analysis of published data. Onco Targets Ther 2014;7:2075-84.

9. Umemura S, Tsubouchi K, Yoshioka H, et al. Clinical outcome in patients with leptomeningeal metastasis from non-small cell lung cancer: Okayama Lung Cancer Study Group. Lung Cancer 2012;77:134-9.

10. Liao BC, Lee JH, Lin CC, et al. Epidermal Growth Factor Receptor Tyrosine Kinase Inhibitors for NonSmall-Cell Lung Cancer Patients with Leptomeningeal Carcinomatosis. J Thorac Oncol 2015;10:1754-61.

11. Zhou C, Wu YL, Chen G, et al. Erlotinib versus chemotherapy as first-line treatment for patients with advanced EGFR mutation-positive non-small-cell lung cancer (OPTIMAL, CTONG-0802): a multicentre, open-label, randomised, phase 3 study. Lancet Oncol 2011;12:735-42.

12. Wu YL, Zhou C, Liam CK, et al. First-line erlotinib versus gemcitabine/cisplatin in patients with advanced EGFR mutation-positive non-small-cell lung cancer: analyses from the phase III, randomized, open-label, ENSURE study. Ann Oncol 2015;26:1883-9.

13. Rosell R, Carcereny E, Gervais R, et al. Erlotinib versus standard chemotherapy as first-line treatment for European patients with advanced EGFR mutation-positive non-small-cell lung cancer (EURTAC): a multicentre, open-label, randomised phase 3 trial. Lancet Oncol 2012;13:239-46.

14. Mok TS, Wu YL, Thongprasert S, et al. Gefitinib or carboplatin-paclitaxel in pulmonary adenocarcinoma. $\mathrm{N}$ Engl J Med 2009;361:947-57.

15. Mitsudomi T, Morita S, Yatabe Y, et al. Gefitinib versus cisplatin plus docetaxel in patients with non-small-cell lung cancer harbouring mutations of the epidermal growth factor receptor (WJTOG3405): an open label, randomised phase 3 trial. Lancet Oncol 2010;11:121-8.

16. Maemondo M, Inoue A, Kobayashi K, et al. Gefitinib or chemotherapy for non-small-cell lung cancer with mutated EGFR. N Engl J Med 2010;362:2380-8.

17. Yang JC, $W u$ YL, Schuler $M$, et al. Afatinib versus cisplatin-based chemotherapy for EGFR mutation-positive lung adenocarcinoma (LUX-Lung 3 and LUX-Lung 6): analysis of overall survival data from two randomised, phase 3 trials. Lancet Oncol 2015;16:141-51.

18. Lehmann U, Bartels S. Liquid biopsy in tumor diagnostics: Applications, perspectives, and limitations of the "cancer liquidome". Pathologe 2019;40:250-5.

19. Crowley E, Di Nicolantonio F, Loupakis F, et al. Liquid biopsy: monitoring cancer-genetics in the blood. Nat Rev Clin Oncol 2013;10:472-84.

20. Wan JCM, Massie C, Garcia-Corbacho J, et al. Liquid biopsies come of age: towards implementation of circulating tumour DNA. Nat Rev Cancer 2017;17:223-38. 
21. Johann DJ Jr, Steliga M, Shin IJ, et al. Liquid biopsy and its role in an advanced clinical trial for lung cancer. Exp Biol Med (Maywood) 2018;243:262-71.

22. Jiang BY, Li YS, Guo WB, et al. Detection of Driver and Resistance Mutations in Leptomeningeal Metastases of NSCLC by Next-Generation Sequencing of Cerebrospinal Fluid Circulating Tumor Cells. Clin Cancer Res 2017;23:5480-8.

23. Huang R, Xu X, Li D, et al. Digital PCR-Based Detection of EGFR Mutations in Paired Plasma and CSF Samples of Lung Adenocarcinoma Patients with Central Nervous System Metastases. Target Oncol 2019;14:343-50.

24. Zhao J, Ye X, Xu Y, et al. EGFR mutation status of paired cerebrospinal fluid and plasma samples in EGFR mutant non-small cell lung cancer with leptomeningeal metastases. Cancer Chemother Pharmacol 2016;78:1305-10.

25. Pentsova EI, Shah RH, Tang J, et al. Evaluating Cancer of the Central Nervous System Through Next-Generation Sequencing of Cerebrospinal Fluid. J Clin Oncol 2016;34:2404-15.

26. De Mattos-Arruda L, Mayor R, Ng CKY, et al. Cerebrospinal fluid-derived circulating tumour DNA better represents the genomic alterations of brain tumours than plasma. Nat Commun 2015;6:8839.

27. Pan $W, \mathrm{Gu} W$, Nagpal S, et al. Brain tumor mutations detected in cerebral spinal fluid. Clin Chem 2015;61:514-22.

28. Ying S, Ke H, Ding Y, et al. Unique genomic profiles obtained from cerebrospinal fluid cell-free DNA of nonsmall cell lung cancer patients with leptomeningeal metastases. Cancer Biol Ther 2019;20:562-70.

29. Yang K, Li J, Zhao J, et al. Developing Ultrasensitive Library-Aliquot-Based Droplet Digital PCR for Detecting T790M in Plasma-Circulating Tumor DNA of Non-smallCell-Lung-Cancer Patients. Anal Chem 2018;90:11203-9.

30. Cheng H, Perez-Soler R. Leptomeningeal metastases in non-small-cell lung cancer. Lancet Oncol 2018;19:e43-55.

31. Li YS, Jiang BY, Yang JJ, et al. Unique genetic profiles from cerebrospinal fluid cell-free DNA in leptomeningeal metastases of EGFR-mutant non-small-cell lung cancer: a new medium of liquid biopsy. Ann Oncol 2018;29:945-52.

32. Kim E, Feldman R, Wistuba, II. Update on EGFR Mutational Testing and the Potential of Noninvasive Liquid Biopsy in Non-Small-cell Lung Cancer. Clin Lung Cancer 2018;19:105-14.

33. Leon SA, Shapiro B, Sklaroff DM, et al. Free DNA in the serum of cancer patients and the effect of therapy. Cancer
Res 1977;37:646-50.

34. Casadio V, Salvi S, Martignano F, et al. Cell-Free DNA Integrity Analysis in Urine Samples. J Vis Exp 2017;(119):55049.

35. Chan LY, Leung TN, Chan KC, et al. Serial analysis of fetal DNA concentrations in maternal plasma in late pregnancy. Clin Chem 2003;49:678-80.

36. Chan MH, Chow KM, Chan AT, et al. Quantitative analysis of pleural fluid cell-free DNA as a tool for the classification of pleural effusions. Clin Chem 2003;49:740-5.

37. Stroun M, Anker P. Circulating DNA in higher organisms cancer detection brings back to life an ignored phenomenon. Cell Mol Biol (Noisy-le-grand) 2005;51:767-74.

38. Jahr S, Hentze H, Englisch S, et al. DNA fragments in the blood plasma of cancer patients: quantitations and evidence for their origin from apoptotic and necrotic cells. Cancer Res 2001;61:1659-65.

39. Gautschi O, Bigosch C, Huegli B, et al. Circulating deoxyribonucleic Acid as prognostic marker in non-smallcell lung cancer patients undergoing chemotherapy. J Clin Oncol 2004;22:4157-64.

40. McGuire AL, Urosevic N, Chan DT, et al. The impact of chronic kidney disease and short-term treatment with rosiglitazone on plasma cell-free DNA levels. PPAR Res 2014;2014:643189.

41. Lui YY, Chik KW, Chiu RW, et al. Predominant hematopoietic origin of cell-free DNA in plasma and serum after sex-mismatched bone marrow transplantation. Clin Chem 2002;48:421-7.

42. Batth IS, Mitra A, Manier S, et al. Circulating tumor markers: harmonizing the yin and yang of CTCs and ctDNA for precision medicine. Ann Oncol 2017;28:468-77.

43. Li BT, Drilon A, Johnson ML, et al. A prospective study of total plasma cell-free DNA as a predictive biomarker for response to systemic therapy in patients with advanced non-small-cell lung cancers. Ann Oncol 2016;27:154-9.

(English Language Editor: J. Gray)

Cite this article as: Liu Y, Yang S, Zhao J, He Z, Ma J, Guo Y, Wang W, Yoshizawa A, Prelaj A, Tiseo M, Normanno N, Van Schil PE, Wang Q, Yang X. Cell-free DNA from cerebrospinal fluid can be used to detect the EGFR mutation status of lung adenocarcinoma patients with central nervous system metastasis. Transl Lung Cancer Res 2021;10(2):914-925. doi: 10.21037/tlcr21-62 


\section{Supplementary}

Table S1 The comparison of EGFR mutation status in the supernatant and sediment of the CSF of all 79 patients

\begin{tabular}{|c|c|c|c|c|c|c|}
\hline & \multicolumn{2}{|c|}{ Supernatant } & Total & $\frac{\text { McNemar's test }}{\mathrm{P}}$ & \multicolumn{2}{|c|}{ Kappa test } \\
\hline Sediment & & & & 0.092 & 0.656 & $<0.001$ \\
\hline Positive & 24 & 3 & 27 & & & \\
\hline Negative & 10 & 42 & 52 & & & \\
\hline
\end{tabular}

The abundance median/quartile of the positive samples of supernatant and sediment was 33.00\% (23.00-49.30\%) and 5.30\% (2.20$15.00 \%)$, respectively. CSF, cerebrospinal fluid.

Table S2 The comparison of EGFR mutation status in CSF and plasma in all 79 patients

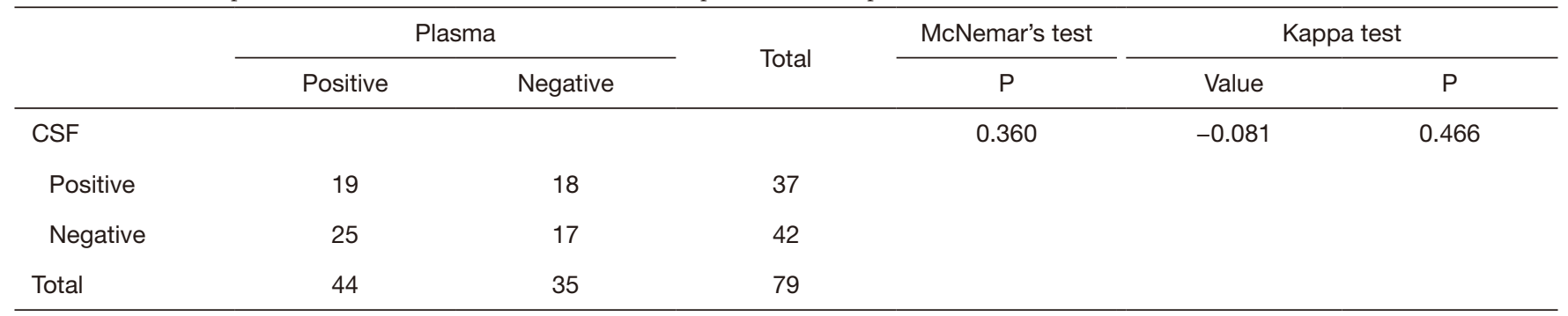

The abundance median/quartile of the positive samples of CSF and plasma was $33.00 \%(16.80-49.30 \%)$ and $2.05 \%(0.67-14.75 \%)$, respectively. CSF, cerebrospinal fluid.

Table S3 The comparison of EGFR mutation status in the CSF and plasma of 53 BM patients

\begin{tabular}{|c|c|c|c|c|c|c|}
\hline & \multicolumn{2}{|c|}{ Plasma } & Total & $\frac{\text { McNemar's test }}{\mathrm{P}}$ & \multicolumn{2}{|c|}{ Kappa test } \\
\hline CSF & & & & $<0.001$ & 0.093 & 0.381 \\
\hline Positive & 11 & 4 & 15 & & & \\
\hline Negative & 23 & 15 & 38 & & & \\
\hline
\end{tabular}

The abundance median/quartile of the positive samples of CSF and plasma was $33.00 \%(11.00-46.70 \%)$ and $2.60 \%(0.90-19.40 \%)$, respectively. BM, brain metastasis; CSF, cerebrospinal fluid.

Table S4 The comparison of EGFR mutation status in the CSF and plasma of 26 LM patients

\begin{tabular}{|c|c|c|c|c|c|c|}
\hline & \multicolumn{2}{|c|}{ Plasma } & Total & $\frac{\text { McNemar's test }}{\mathrm{P}}$ & \multicolumn{2}{|c|}{ Kappa test } \\
\hline CSF & & & & 0.004 & -0.061 & 0.606 \\
\hline Positive & 8 & 14 & 22 & & & \\
\hline Negative & 2 & 2 & 4 & & & \\
\hline
\end{tabular}

The abundance median/quartile of the positive samples of CSF and plasma was $31.80 \%(16.80-55.00 \%)$ and $1.27 \%(0.37-5.08 \%)$, respectively. LM, leptomeningeal metastasis; CSF, cerebrospinal fluid. 
Table S5 The comparison of EGFR mutation status in the CSF and plasma of 26 patients without neurological symptoms

\begin{tabular}{|c|c|c|c|c|c|c|}
\hline & \multicolumn{2}{|c|}{ Plasma } & Total & $\frac{\text { McNemar's test }}{\mathrm{P}}$ & \multicolumn{2}{|c|}{ Kappa test } \\
\hline CSF & & & & NA & NA & NA \\
\hline Positive & 3 & 0 & 3 & & & \\
\hline Negative & 15 & 8 & 23 & & & \\
\hline
\end{tabular}

The abundance median/quartile of the positive samples of CSF and plasma was $40.00 \%$ and $2.20 \%(0.90-9.90 \%)$, respectively. CSF, cerebrospinal fluid.

Table S6 The comparison of EGFR mutation status in the CSF and plasma of 53 patients with neurological symptoms

\begin{tabular}{|c|c|c|c|c|c|c|}
\hline & \multicolumn{2}{|c|}{ Plasma } & Total & $\frac{\text { McNemar's test }}{\mathrm{P}}$ & \multicolumn{2}{|c|}{ Kappa test } \\
\hline CSF & & & & 0.185 & -0.051 & 0.697 \\
\hline Positive & 16 & 18 & 34 & & & \\
\hline Negative & 10 & 9 & 19 & & & \\
\hline
\end{tabular}

The abundance median/quartile of the positive samples of CSF and plasma was $32.10 \%(15.00-49.30 \%)$ and $2.00 \%(0.63-19.50 \%)$, respectively. CSF, cerebrospinal fluid.

Table S7 The comparison of EGFR mutation status in the CSF and plasma of 29 EGFR-TKI-naïve patients

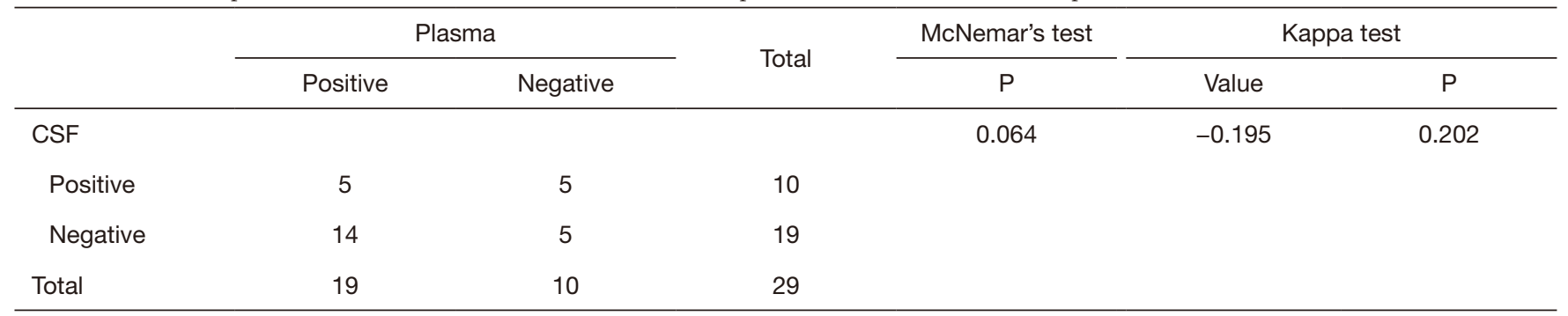

The abundance median/quartile of the positive samples of CSF and plasma was $4.90 \%(3.70-33.80 \%)$ and $2.80 \%(0.60-20.50 \%)$, respectively. CSF, cerebrospinal fluid; TKI, tyrosine kinase inhibitor.

Table S8 The comparison of EGFR mutation status in the CSF and plasma of 50 EGFR-TKI-treated patients

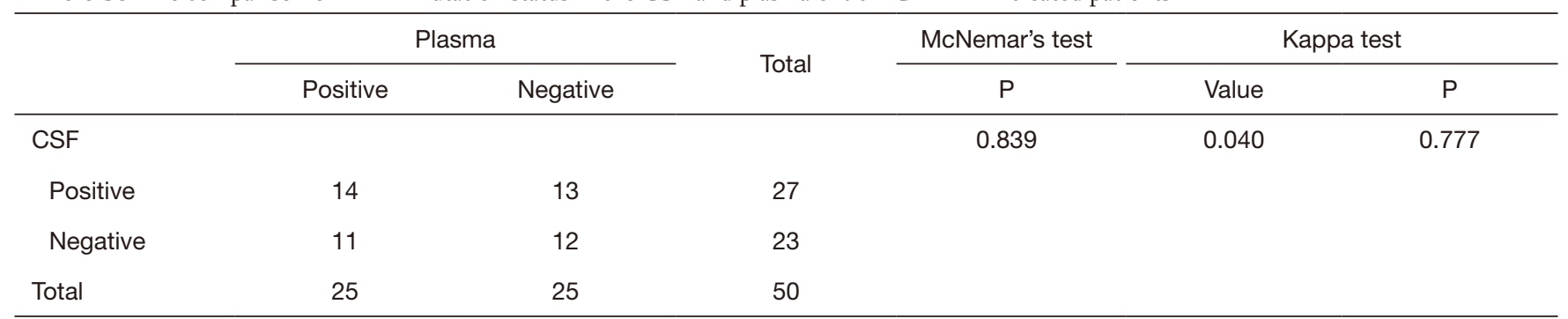

The abundance median/quartile of the positive samples of CSF and plasma was $33.50 \%$ (26.65-52.15\%) and 2.00\% (0.70-10.60\%), respectively. CSF, cerebrospinal fluid; TKI, tyrosine kinase inhibitor. 
Table S9 The comparison of EGFR mutation status in the CSF and plasma of 39 patients with EGFR exon 19 deletion*

\begin{tabular}{|c|c|c|c|c|c|c|}
\hline & \multicolumn{2}{|c|}{ Plasma } & Total & $\frac{\text { McNemar's test }}{\mathrm{P}}$ & \multicolumn{2}{|c|}{ Kappa test } \\
\hline CSF & & & & 0.263 & -0.034 & 0.821 \\
\hline Positive & 13 & 7 & 20 & & & \\
\hline Negative & 13 & 6 & 19 & & & \\
\hline
\end{tabular}

The abundance median/quartile of the positive samples of CSF and plasma was $33.00 \%$ (16.40-43.85\%) and 1.95\% (0.70-9.90\%), respectively. *, two patients had both L858R and E19Del EGFR mutation. CSF, cerebrospinal fluid.

Table S10 The comparison of EGFR mutation status in CSF and plasma in 42 patients with EGFR exon 21 L858R mutation*

\begin{tabular}{|c|c|c|c|c|c|c|}
\hline & \multicolumn{2}{|c|}{ Plasma } & Total & $\frac{\text { McNemar's test }}{\mathrm{P}}$ & \multicolumn{2}{|c|}{ Kappa test } \\
\hline CSF & & & & 1.000 & -0.153 & 0.320 \\
\hline Positive & 7 & 12 & 19 & & & \\
\hline Negative & 12 & 11 & 23 & & & \\
\hline
\end{tabular}

The abundance median/quartile of the positive samples of CSF and plasma was $29.60 \%$ (15.00-49.30\%) and 2.40\% (0.57-10.60\%), respectively. *, two patients had both L858R and E19Del EGFR mutation. CSF, cerebrospinal fluid.

Table S11 The comparison of EGFR mutation abundance in CSF and plasma

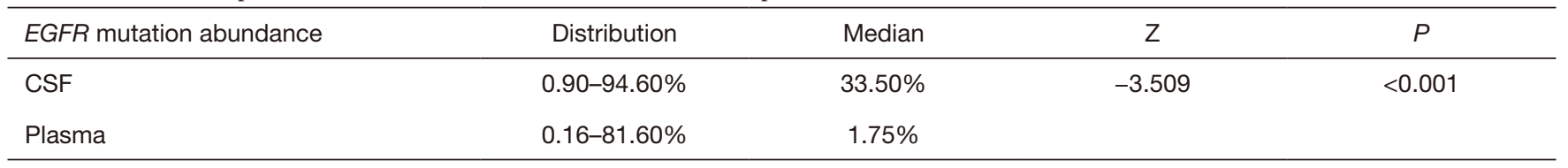

CSF, cerebrospinal fluid. 